\title{
Sexual Relationship, Power and Safe Sex Practices among Female Bar Workers in Kinondoni Municipality, Dar es Salaam, Tanzania
}

\author{
Mangi Job Ezekiel, Judith Narlis Metta, Idda Hubert Mosha
}

\section{ABSTRACT}

Background: HIV and AIDS continue to challenge the health sector and economies in low and middle-income countries. Effectiveness condom use in preventing transmission of $\mathrm{HIV}$ is jeopardized due to recent reported decline in condom use. Alcohol serving venues are meeting points for sex partner which is a significant determinant of risk sexual practices while gender inequities impose challenges to women in negotiating safe sex.

Methods: Cross sectional exploratory study was conducted which deployed a qualitative method in data collection. Purposive sampling was used to select fourteen study participants. In-Depth Interview guide was used to gather information from female bar workers on perceptions and experiences in sexual relationships that are initiated following their interaction with male customers in alcohol serving settings and their negotiation for safe sex. This study explored sexual relationship power of Female Bar Workers (FBWs) in safe sex negotiations as they interact in alcohol serving venues in Kinondoni municipality in Dar es Salaam, Tanzania. Data was analysed manually by using thematic approach.

Results: Negotiation ability and sexual relationship power continue to be a key determinant of safe or unsafe sexual practices in sexual relationships of female bar workers. Although power to negotiate and make decisions towards safe sex was widely reported by female bar workers when they engage in casual sexual relationships, such power is outweighed by their own willingness to engage in multiple sexual relationships to supplement their meagre incomes.

Conclusion: Health promotion should continue to focus on behaviour change interventions with consideration of gendered roles and power in sexual relationships.

Keywords: Female bar workers, power, sexual relationship, Tanzania.
Submitted : November 24, 2021

Published : January 13, 2022

ISSN: 2593-8339

DOI: $10.24018 /$ ejmed.2022.4.1.1153

M. J. Ezekiel

Department of Behavioural Sciences, School of Public Health and Social Sciences, Muhimbili University of Health and Allied Sciences, Dar es Salaam, Tanzania.

(e-mail: e_mangi@yahoo.com)

J. N. Metta

Reaching the Unreached Tanzania (RUT), Dodoma, Tanzania.

(e-mail: judmeta@gmail.com)

I. H. Mosha*

Department of Behavioural Sciences, School of Public Health and Social Sciences, Muhimbili University of Health and Allied Sciences, Dar es Salaam, Tanzania.

(e-mail: ihmosha@yahoo.co.uk)

*Corresponding Author

\section{INTRODUCTION}

Despite the documented substantial burden of the HIV and AIDS pandemic in sub-Saharan Africa, there has been an alarming decrease in condom use or an increase in the number of sexual partners among the general population. Such situation is likely to jeopardize documented achievements along the decline in new HIV infections and prevalence between 2001 and 2011 [1], [2].

Available evidence suggests that, there is no direct scientific causal relationship between alcohol and transmission of Sexually Transmitted Infections (STIs) including HIV [3]. However, some studies show the link between alcohol use and incidence of STIs particularly HIV by considering additional facts that alcohol use can highly interfere with cognitive aspects of an individual's decisionmaking ability which may lead to risky health behaviours such as unsafe sex practices and involvement with multiple sexual partners [1], [4]-[6].

Similarly, alcohol serving venues are well known places where social networks are formed including between those who are serving alcohol and consumers. The venues act as meeting points for sexual partners as significant determinants of risk sexual practices such as multiple sexual partners and/or lower levels of condom use, both of which foster the transmission of STIs and HIV [7]. In light of that, it is documented that $35 \%$ of female bar workers (FBWs) in Tanzania have actively been engaging in sex with the male customers consuming alcohol in the alcohol serving venues [8]. Another study conducted in northern Tanzania shows that; higher levels of alcohol consumption have been observed among FBWs compared to other females in the general population, a behaviour that is influenced by their regular interaction with customers in alcohol serving venues [9].

Sexual relationship power refers to the ability or skills that a person (man or woman) can exercise to influence or 
control actions of another person in the context of relationship between men and women such as when making decisions on sex or condom use. In the context of this study sexual relationship power is considered as the basic component that determines women's ability to negotiate safe sex practice [10].

Safe sex practice is a phrase often used to refer to a combination of acts when practicing sex (vaginal, anal or oral) that leaves a person at a lower risk of sexually transmitted infections (STIs) including HIV [11]. Practicing safe sex is considered a challenge that impedes realization of health prevention interventions focusing on mitigation transmission of STIs including HIV in Tanzania. According to the Tanzania Demographic Health Survey [12] among people who had multiple sexual partners, only $24 \%$ of men and $27 \%$ of women reported to have practiced safe sex by using condom the last time they practiced sex. In the same scenario, safe sex practices among never married women and men is still far from being a consideration as it is documented that only a half of them reported to have practiced safe sex by the use of condom during their last sexual activity. However, despite efforts encouraging safe sex practices that have been documented by the Ministry of Health Tanzania [13] to show an increase in condom use during the last sexual activity among men from $49 \%$ to $59 \%$ and from $46.3 \%$ to $58 \%$ among women, it is also documented that safe sex practices among unmarried women is still very low with only $15 \%$ reported to have used male condoms [14].

Avert [15] reported that women are severely hampered by HIV in Tanzania due to different reasons, including the fact that they experience serious difficulties in negotiating safer sex when they enter into sexual relationships because of gender inequalities. Furthermore, HIV remains to be a big problem in the country, with a prevalence rate of $6.5 \%$ among women and 3.5\% among men in 2017 [16].

HIV prevalence among FBWs in Tanzania varies within the regions. In 2000, it was estimated to be around 50\% in Dar es Salaam [17] while in 2014 the estimations in Mbeya were at $60 \%$ and in Moshi at $26 \%$ [18]. It is also documented that, women experience difficulties to negotiate safe sex due to male dominance in decision making such as when it comes to decision making over condom use [19] and in family planning issues and number of children to have [20]. According to [21] gender inequities pose a great challenge to HIV prevention through its impact on sexual relationships. Recent data in sub-Saharan Africa show that income disparity and gender inequality are among the key issues that drive the pandemic in the region where unequal gender norms that limit the agency and voice of women and girls, reduce their access to education and economic resources contribute to the higher HIV risk among women in HIV prevalence areas [22]. In the context of this study, sexual relationship power denotes the ability of an individual (man or woman) to influence decisions regarding safe sex practices.

Evidence shows that women comprise a large proportion of the employment category in accommodation, food and alcohol service activities in Tanzania [8]. The role of gender and how it relates to social norms and inequalities provides the basis to understanding risk behaviours between men and women especially in the context of sexual relationships. Alcohol serving locations have been widely documented to play a momentous role in influencing risky sexual behaviors such as unsafe sex practices and multiple sexual partnerships [7], [23]-[25].

Reviewed literatures show that there are a number of studies which examined the influence of sexual relationships and safe sex practices. However, the review showed that most of these studies have been conducted among different population groups and in different settings out of Tanzania. Additionally, majority of the studies documenting sexual and relationship power and sexual practices are quantitative [1], [19].

Moreover, several studies have documented on sexual relationship power and safe sex practices and some of them have tried to establish relationship between males and females and how one influences another. Decisions making power and negotiation power have been documented as the key factors determining safe or unsafe sexual practices among couples [19]. Nonetheless, little is known about FBWs safe sex practices in their sexual relationships that are initiated in the context of alcohol venues hence the need to explore FBWs ability to negotiate and practice safe sex within such sexual relationships.

Therefore there is limited qualitative evidence on how the concept of sexual relationship power and how it relates to female bar workers to inform strategies to address HIV in this populace. Evidence shows that FBWs are at higher risk of STIs and HIV due to their work environment where sexual relationships tend to emerge and alcohol being the main business item [26]-[27]. Moreover, nature of FBWs occupation has been generally reported to influence their high alcohol consumption [28] compared to other females in general population.

Therefore, the aim of this study was to explore how sexual relationship power dictates decision making with regard to safe sex practices of an individual FBW. Specifically, the objectives of this paper are twofold: first, to assess sexual relationship, power among FBWs, second, to explore safe sex practices among FBWs in Dar es Salaam, Tanzania. The findings of the present study are expected to inform strategies of HIV prevention interventions that target populations at increased risk including those in alcohol serving places.

\section{Materials AND Methods}

\section{A. Study Design and Context}

This was a cross sectional exploratory study. This study adopted phenomenological approach to explore FBWs experience of their alcohol use and sexual relationships as they interact with male clients in alcohol serving and drinking environments. Data were generated through indepth Interviews (IDI) guide with selected female bar workers.

\section{B. Study Area}

The study was conducted in Dar es Salaam Kinondoni municipality (recently divided into Kinondoni and Ubungo) which is located on the eastern part of Tanzania being one of the then three municipalities of the Dar es Salaam city, 
together with Ilala and Temeke (recently sub divided into Temeke and Kigamboni). Kinondoni district has many alcohol bars compared to other district that is why it was selected for this study. Dar es Salaam region has an estimated population of $4,364,541$ with a population growth rate at 5.6, the main source of cash income being sale of goods purchased from others for resale. The population of Kinondoni municipality was estimated to be $1,775,049$ in 2012 of which males were 860,802 , females 914,247 . The rate of women involvement in decision making is at $29.0 \%$ considering posts occupied by women in managerial, professional and political spheres being lower compared to Ilala and Temeke districts which have higher rates at $36.6 \%$ and $33.4 \%$ respectively [29].

\section{Sampling Procedures}

Purposeful sampling was used to select the study participants. Purposive sampling allowed the researcher to select the sample that could facilitate rich description and understanding of the phenomena under investigation based on the study topic. Moreover, purposive sampling for maximum variations of study participants enabled us to hear different opinions on the sexual relationship power and safe sex practices of FBWs. A total number of seven licensed bars were selected purposeful. From each bar two FBWs who were 18 years and above and have worked in bars in Dar es Salaam city for more than six months prior this study were selected to participate in this study. The total number of the respondents was 14 following a saturation of the information obtained in the process of data collection. FBWs were approached during their working hours and briefed about the study. Appointment for interviews was scheduled with those who voluntarily agreed to participate in the study and the interviews were conducted in their convenient time and location outside the working hours and place respectively. With consent from study participants all in depth interviews were digitally recorded.

\section{Data Collection Tool}

In depth interview guide was used to collect data from study participants. A total of fourteen study participants were purposefully selected to participate in this study. The interview guide was originally prepared in English before being translated into Kiswahili by the PI. The PI conducted in-depth interviews in Kiswahili, a language in which all the informants were competent. Each interview session lasted half an hour. Two IDIs were executed each day. This facilitated reflection on, and consolidation of, emerging issues for further questioning. The interview guide focused on assess sexual relationship, power among FBWs and safe sex practices among FBWs. Conducive places were secured to provide privacy and free conversation between the PI and the informants. All IDIs were digitally recorded, after seeking consent from the participants.

\section{E. Data Management and Analysis}

The audio files were uploaded into a password protected computer to ensure data security was maintained throughout data management; storage and use. The digitally-recorded interviews were transcribed and translated into English and thereafter back translated into Kiswahili.

Thematic analysis using five stages according to Braun and Clarke was performed to establish meaningful patterns: First step was familiarization with the data, generating initial codes, searching for themes among codes, reviewing themes and presenting the results [30]-[31]. The first author analysed the data manually by initiating the coding and category assignments. Then the second and third authors went through the data identifying discrepancies. The discrepancies were discussed and consensus was reached after referring to the recorded interviews. Codes and categories that emerged from data were later sorted out to form the main themes that emerged, as presented in the findings section.

\section{F. Ethical Considerations}

We obtained a research permit from the ethics committee of the University of Dar es Salaam. We also obtained permission to conduct the study from the regional, district, wards and streets authorities. Individual informed written consents were sought and obtained from the study participants prior to their participation in the study. All information was kept confidential, with names excluded from the recorded materials to avoid giving away the identity of the participants.

\section{FINDINGS}

\section{A. Characteristics of Study Participants}

A total of fourteen (14) female bar workers participated in this study. Participants who consented to participate in the study were between the age of nineteen (19) and thirty-eight (38). Seven participants completed primary school, 5 completed ordinary secondary school and 2 did not complete primary school. All participants reported to be actively engaging in sexual practices and one reported being formally married at the time of data collection. One was married, one was a widow, five participants reported to be separated from their partners while seven reported to be single but sexually active. Ten (10) participants reported to be living and caring for at least one child at their households.

\section{B. Experiences of Female Bar Workers on Alcohol Serving Job}

Majority of study participants reported to have been working between one year and five years of experience working as FBWs in different alcohol serving points. Moreover, they reported that they started working as FBWs following life challenges ranging from failure to continue with school to sexual relationships break-offs that rendered them with no alternative ways of earning income.

"I searched for a job but I was not able to get any, one day I met a woman and she told me that there was a vacancy for female bar workers somewhere. I told her that I had never done such kind of a job since I was born, but I had to do it because I was in a need of job" [IDI, FBW 8].

The explanations from respondents indicate that none of the female bar workers reported to like their work. All participants declared that they don't like the kind of work they do but they just do it because they need money to 
sustain their lives and have no other alternatives.

"I don't like to be a bar maid, but I am doing it because of my life situation, I had no other option to earn income" [IDI, FBW 6].

Participants also reported about their encounters of being seduced by male customers. All participants declared to have been approached by male customers who wanted them for sex and could further explain how frequent male customers have been approaching them. Again, all participants reported to have been approached for sex by male customers every day and sometimes in a countless time in a single working day.

"I have been approached for sex too much by men, and I cannot even count the number of men who approach me in a day or week" [IDI, FBW 9].

\section{Relationship Stability}

Majority of study participants reported to be in casual sexual relationship. However, they reported that their sexual relationship doesn't last long and that it could take less than six months. They narrated that such relationships are associated with FBWs' concerns that, such male sexual partners who initiate and enter into sexual relationships with them are searching for temporary sexual satisfaction, while the FBWs reported to be into casual sexual relationship for the purpose of earning more money to supplement their insufficient income.

"Most of our sexual relationships do not last for more than a month, those who last in their relationship for more than a month are few, though there are others (FBWs) who have partners for a long time but most of them not last for more than a month ... if you have a partner for more than a month ... you say 'thank you, God'...” [IDI, FBW1].

Majority of participants reported to have no stable sexual relationships. Having multiple sexual relationships was noted to be a dominant feature among FBWs. Majority of them reported that they have multiple sexual partners because they needed financial support. They further recounted those sexual relationships that they engage themselves into which are initiated at the alcohol serving and drinking point do not have any future.

"...there is another man I went to school with, I am still in a sexual relationship with him [...] it doesn't mean that I don't have other [men] it's because he is at school [...] he is now at form five level, he cannot send me money for daily needs since he is also a dependent at his home ..." [IDI, FBW 4].

Study participants reported that majority of men who seduce them at alcohol serving avenues have expectations of getting sex workers there. They recounted that some of their male customers can seduce bar workers but then may end up getting a sex worker instead. The sex workers may be taken for sex at nearby hotels or guest houses.
"A male customer may seduce me then at the same time, and he may call a female sex worker and go with her to a nearby guest house ... then he comes back later and continue seducing me ... most of them come with their intention to get prostitutes here ..." [IDI, FBW2].

\section{Sexual Relationship}

Two types of multiple sexual relationships were reported by study participants. The first one is having a regular sexual partner whereby a FBW enters into a sexual relationship with a man while she is well aware that the man has a committed relationship already i.e. having a wife.

“...sometimes it happens you have already fallen in love (if you ask about his marital status) he tells you 'That woman is the one disturbing me, I don't even know her' sometimes I see it clearly that he is lying, I forget about that but I clearly know he has a wife ..." [IDI, FBW11].

The second type of multiple relationships was a causal relationship, whereby FBWs can just enter into a sexual relationship with males who are customers at alcohol serving points for the purpose of earning money in return for sex, and this was reported to be a dominant kind of sexual relationships among study participants.

"As for me I do not have any plans with males who seduce me at my place of work. I accept to be with them because they help me money to buy small things, so before I say yes to them I usually ask them to support me financially and they may say yes or no...so for those who say yes I might agree to be into sexual relationship with them" [IDI, FBW12].

\section{E. FBWs' Perception of Condom Use among Male Sexual Partners}

Majority of FBWs narrated their experience of proposing condom use with their sexual partners. They reported that majority of their sexual partners do not accept condom use especially when you have been in a sexual relationship for a long time with them. FBWs were of the view that majority of male customers who seduce them just think about protecting themselves against HIV and forget the fact that there are other sexually transmitted diseases as well. They narrated that because men are stronger than women, they can sometimes force them to have sex unsafe sex with them.

"Majority of male customers who seduce me say that they can't have sex with a condom and force me to have sex with them without condom; sometimes they can say that we have been in a relationship with me for so long, so we know each other enough and therefore no need of using the condom. You know people don't think of other sexual transmitted diseases they just know HIV/AIDS only. You know that men can force you to have sex without a condom as you know they are strong and powerful so they can use force ...it happened once to me..." [IDI, FBW 8].

\section{DISCUSSION}

This study explored sexual relationship power and 
condom use among female bar workers. Central to the issues sexual relation power is how ideas around gender and power have been applied to understand how sexual relationship power determines condom use. Sexual division of labour sexual division of power and social norms which are related to gender roles were examined. In addition, the concepts of sexual relationship particularly relationship control and decision-making dominance were observed.

Male partners were reported to be the controllers of relationships and the decision influencers on matters pertaining to condom use. It has also been revealed that, sexual inequity and power imbalance in sexual relationships continue to have impact on condom use and safe sex practice as a whole.

Sexual division of labour (socio-economic risk factors) is one area that pictures partners' power for negotiating safe sex. FBWs have been assigned and are performing jobs that yielded very low income and due to that they find themselves engaging in higher risk behaviours since their needs for money outweigh their ability to negotiate for safer sex options hence not courageous enough to practice safe sex such as using condom. In relation to findings elsewhere, it was observed that female bar workers have been entering into sexual relationships for different reasons, one of the reasons being to earn additional incomes particularly from the male customers -a situation that leaves them in multiple sexual relationships [8], [32]. Therefore, circumstances force them to do so mainly because the incomes from their job as bar workers are not sufficient enough to fulfil their basic needs.

Despite of the fact that female bar workers have shown a certain level of courage and ability to negotiate safe sex in casual relationships, it was commonly reported that when male partners refused to use condom, female bar workers had no ability to postpone or rather escape unsafe sex practices. This is contrary to a study done by [10] whereby a majority $(70 \%)$ of female participants reported that they could refuse having sex if their male partners refused using condom. The inconsistency noted between these two studies might be due to the differences in study settings and prevailing gender norms. Reference [10] conducted their study in the United States of America where economic status and awareness of women rights is higher compared to Tanzania, a situation that creates an obvious gap between the two settings.

The findings reported that to express unwillingness to use condom, male partners could use physical force over their female partners as an option to have sex as a result female bar worker could lose the courage to negotiate safe sex and leaving their partners to decide to do whatever they want and feel to do. This implies that physical force is one of the key predictors of regular safe sex practices. This is consistent with previous studies [19], [35] whereby participants who ever suffered multiple types of violence were vulnerable to unsafe sex practices activity. This shows that, a woman who has ever suffered abuse is likely to lose power in making decision especially when it comes to safe sex practice if their partners do not support safe sex practices such as condom use. Therefore, such women are more likely to participate in unsafe sex practices for the fear and failure to negotiate with their sexual partner(s).
FBWs with reported power in sexual relationship in this study were those who reported to be in casual relationships and were more likely to have the ability to negotiate safe sex with their partners. However, having no committed sexual partners alone cannot be concluded to enable FBWs have influence and decide what they desire to be safe considering that the intentions of the sexual relationship they engage into is based on the material needs a situation that leaves them lacking fully control over the decisions they would otherwise desire in such sexual relationships.

\section{A. Limitations}

This study explored power in sexual relationships of FBWs in Kinondoni Municipality based on their experience working and interacting in alcohol serving environment during a specific period in 2019 and therefore, the findings and conclusions must be interpreted in that context of space and time. The study relied on self-reports from FBWs and there are possibilities of recall and information bias. However, the researchers took great care in selecting the timing and places of interviews to ensure that the study participants were comfortable to offer their experiences.

A concern of social desirability bias was anticipated from respondents especially from FBWs who were likely to provide information that they believed was what the interviewer wanted to hear rather than revealing what actually happens in terms of their personal behaviours. It was vivid that being FBWs and identified as so led some respondents to deny engaging in risky sexual behaviour such as unsafe sex practices. However, care was taken by employing research techniques such as proper rapport building with study participants and probing throughout the interviews which enabled to counter such limitations and respondent revealed some issues that they had explained otherwise.

\section{CONCLUSION}

The findings revealed that, these sexual relationships among FBWs are characterized by gendered power dynamics. FBWs have indicated their capability to express sexual agency; on but they become passive depending on the type of sexual relationship they have. Money was the motive mentioned by majority of FBWs to engage in sexual relationships with their customers. FBWs perceive themselves to be less powerful in sexual relationships, FBWs negotiated safe sex in their temporary or casual relationships over permanent sexual relationships something which put them at risk of unsafe sex. Therefore, health behaviour change interventions need to tailor messages around promoting sexual relationship power to address gender norms that perpetrate inequalities and increasing risk of sexually transmitted infections. In line with empowering FBWs with information and sexual negotiation skills, these specific efforts should aim at economic empowerment.

\section{ACKNOWLEDGMENT}

We acknowledge all study participants for their time and sharing information with us. 


\section{CONFLICT OF INTEREST}

Authors declare that they do not have any conflict of interest.

\section{REFERENCES}

[1] Nkosi S, Rich EP and Morojele NK. Alcohol Use, Sexual Relationship Power and Unprotected Sex among Patrons in Bars and Taverns in Rural Areas of Northwest Province, South Africa. AIDS Behavior. 2014.

[2] UNAIDS. Global Report: UNAIDS Report on Global AIDS Epidemic. 2013.

[3] ICAP. Drinking and HIV/AIDS Risk. ICAP Health Briefings. ICAP International Centre for Alcohol Policies. Analysis. Balance. Partnership. 2012.

[4] Chersich MF, Luchters SM, Malonza IM, Mworogo P, King'ole N and Temmerman M. Heavy episodic drinking among Kenyan female sex workers is associated with unsafe sex, sexual violence and sexually transmitted diseases. International Journal of STD and AIDS. 2007; 18: $174-813$.

[5] Fisher JC, Bang H and Kapiga SH. Association between HIV infection and Alcohol use: A systematic review and Meta-Analysis of African Studies. American Sexually Transmitted Diseases Association. 2007.

[6] Standerwick K, Davies C, Tucker L and Sheron N. Binge drinking, Sexual behavior and Sexually Transmitted Infection in the UK. International Journal of Sexually Transmitted Diseases. 2007; 18: $810-813$.

[7] Kalichman SC. Social and Structural HIV Prevention in AlcoholServing Establishments. Review of International Interventions Across Populations. Alcohol Research and Health. 2010; 33(3).

[8] Harling G, Muya A, Ortblad KF, Mashashi I, Dambach P, Ulenga N, et al. HIV risk and pre-exposure prophylaxis interest among female bar workers in Dar es Salaam: Cross-sectional Survey. BMJ Open. 2019.

[9] Mongi AS, Baisley K, Ao TT-H, Chilongani J and Aguirre-Andreasen A, et al. Factors Associated with Problem Drinking among Women Employed in Food and Recreational Facilities in Northern Tanzania. PLoS One. 2013; 8(12).

[10] Pulerwitz J, Gortmaker SL, DeJong W. Measuring sexual relationship power in HIV/STD research. Sex Roles. 2000; 42(7-8): 637 - 660.

[11] Aids Map. Unprotected Sex. NAM Publications, UK. 2014.

[12] United Republic of Tanzania. Tanzania demographic health survey. Dar es Salaam, Tanzania: National Bureau of Statistics (NBS) and ICF Macro; 2010.

[13] Ministry of Health (MoH). Global AIDS Response Country Progress Report. 2014.

[14] United Republic of Tanzania. Tanzania demographic health survey \& Malaria Indicator Survey. Dar es Salaam, Tanzania: National Bureau of Statistics (NBS) and ICF Macro; 2015-16.

[15] Avert. HIV/AIDS and Tanzania. [Internet] 2015. [cited 2017 September 24] Available on: http://www.avert.org/hiv-aidstanzania.htm/.

[16] Ministry of Health (MoH) Tanzania. [Internet] 2017. [cited 2016 December] DeTanzania HIV Impact Survey 2016-2017Summary Sheet. Available from: https://phia.icap.columbia.edu/wpcontent/uploads/2017/ 11/Tanzania_SummarySheet_A4.English.v19.pdf

[17] Mbaga, FST. Women Sexual Empowerment: Adoption of FemaleControlled Preventive Measures Against Transmission of HIV Infection: A case study; Perception of Female Condom As a means for Sexual Empowerment for Protection Against HIV Infection Among Barmaids, Kinondoni Municipality, Dar es Salaam Region Tanzania. 2000.

[18] URT. HIV and STI Biological and Behavioral Survey, 2013: A study of Female Sex Workers in seven Regions: Dar es Salaam, Iringa, Mbeya, Mwanza, Shinyanga, Tabora and Mara. Ministry of Health and Social Welfare, National Programme (NACP). AIDS Control. 2014.

[19] Matsuda Y, McGrath JM, Jallo N. Use of the Sexual Relationship Power Scale in Research: An Integrative Review. Hispanic Health Care International. 2012; 10(4).

[20] Mosha I, Ruerd R, Kakoko D. Family planning decisions, perceptions and gender dynamics among couples in Mwanza, Tanzania: a qualitative study. BMC. Public Health. 2013; 13: 523
[21] Pettifor AF, Measham DM, Rees HV, Padian NS. Sexual Power and HIV Risk, South Africa. International Conference on Women and Infectious Diseases. 2004; 10: 11.

[22] UNAIDS. Miles to Go. Closing Gaps, Breaking Barriers, Righting Injustices. 2018.

[23] Bryant KJ, Nelson S, Braithwaite S, Roach D. Integrating HIV/AIDS and Alcohol Research. Alcohol Research \& Health. 2010; $33: 3$.

[24] Campbell ANC, Tross S, Dworkin SL, Hu M, Mannuel J, Pavlicova $\mathrm{M}$, et al. Relationship Power and Sexual Risk among Women in Community-Based Substance Abuse Treatment. Journal of Urban Health. 2009; 86: 6.

[25] Kalichman SC, Eaton L, Cherry C. Sexually transmitted infections and infectiousness beliefs among people living with HIV/AIDS: Implications for HIV treatment as Sex Workers in Southern Tanzania. Baltimore, Maryland. Prevention. HIV Med. 2010; 11(8): 502-509.

[26] Mwendah HK, Mallya ET. HIV/AIDS and Public Administration: Tanzania Country Foresight Paper. 2014.

[27] Messersmith L, Beard J, Agyarko-Poku T, Longobardi D, Asafo M, Corneliess $\mathrm{C}$, et al. "I can decide to use the property I have to make money": HIV Vulnerability of Bar Workers and Bar Patrons in Kumasi, Ghana. Research Report - Operations Research among Key Populations in Ghana. 2014.

[28] Akarro RRJ. Some Factors Associated with High Risk Behavior Among Bar Maids in Tanzania. Current Research Journal: Soc. Sci. 2011;3(3): 207-212.

[29] Exavery A, Kamte MA, Jackson E, Noronha J, Sikustahili G, Tani K, et al. Role of condom negotiation and condom use among women of reproductive age in three districts in Tanzania. 2012.

[30] Clarke V, Braun V. Teaching thematic analysis: overcoming challenges and developing strategies for effective learning. Psychologist. 2013; 26 (2): 120-3.

[31] Braun V, Clarke V, Hayfield N, Terry G. Thematic analysis. In: Handbook of Research Methods in Health Social Sciences. 2019: $843-60$.

[32] Beckham SW. 2013. "Like any other woman"? Pregnancy, motherhood and HIV among Sex Workers in Southern Tanzania. Baltimore, Maryland.

[33] Swan, J. 2016. Sex, Power, and Risk; Who Is Really In Control When It Comes To Sex? [Internet] 2016. [cited 2018 October 14] Available from: https://www.psychologytoday.com/us/close.

[34] Peixoto ER, Barros FC, Guimaraes. Factors associated with unprotected sexual practice among men and women with mental illnesses in Brazil. Cad Saude Publica. 2014; 37(7): 1475-86.

[35] Dunkle K, Jewkes R, Brown H, Mclntye J, Gray G, Harlow S. Gender-based violence and HIV infection among pregnant women in Soweto. A technical report to Australian Agency for International Development. 2003.

[36] Gupta R. Gender, Sexuality and HIV/AIDS: The what, the why and the HIV. Washington DC: ICRW. 2000.

[37] Chege J. Interventions linking gender relations and violence with reproductive health and HIV: Rationale effectiveness. Agenda Special Focus. 2005. 